worked out, however, and Administration officials suggest that it will be weeks, if not months, before it is.

As for the plan to build a large centrifuge enrichmenmt plant, this again is coupled with Carter's moves to curb nuclear proliferation. In 1974, the United States closed its order books for long-term supplies of enriched uranium to other countries because of projections that US enrichment capacity would be used up. The move is said to have prompted many countries to seek alternative sources of supply, and it may have been a key factor in persuading Brazil to negotiate the purchase of an enrichment plant from West Germany. Carter has therefore announced that the United States will re-open its order books, and that a supply of enriched uranium will be guaranteed to those countries which renounce the development of nuclear weapons.

To meet the projected demand, a large centrifuge enrichment plant will be constructed at a cost of some $\$ 4,400$ million. So far, all US enrichment has used the gaseous diffusion process, and previous plans called for expansion of an existing diffusion plant at Portsmouth, Ohio. Administration officials say, however, that the gas centrifuge process, which has been developed in secrecy at Oak Ridge, Tennessee, is now ready to be commercialised. So far, however, no site for the new plant has been chosen, though hints that it may go to Oak Ridge, to compensate for cancellation of the breeder reactor project there, have sparked loud protests in Portsmouth.

In addition to relying on a modest expansion of nuclear generating capacity, Carter's plans for medium-term energy supply also envisage considerable expansion in the use of coal. $\mathrm{He}$ has proposed measures to force industry to convert from oil and gas to coal, announced large increases in research and development efforts aimed at producing synthetic oil and gas from coal, and recommended a number of regulations to reduce the environmental consequences of such an expansion.

There is, however, one potential environmental problem which could prove intractable: the so-called greenhouse effect which may lead to changes in the global climate from large increases in atmospheric carbon dioxide. Carter announced last week that a major study of the greenhouse effect, costing $\$ 3$ million, will be launched this year.

Carter's energy plan will now be picked over on Capital Hill, and already special interest groups are clawing at it. The debate is likely to rage for the rest of this year.

GEOS

\title{
Hope from despair?
}

Stuart Sharrock in Washington and Chris Sherwell in London report on the ill-starred launch of Geos, the European Space Agency's scientific satellite

ON Tuesday of this week a group of space scientists and another of space engineers met separately on opposite sides of the Earth to discuss an event that occurred above the globe halfway between them. Last Wednesday, twenty minutes after a Thor-Delta 2914 rocket had taken off from Cape Canaveral only 0.2 seconds later than its scheduled lift-off of 05.15 , the hopes of 11 research institutes from eight member countries of the European Space Agency turned to despair as a malfunction during the separation of the second and third stages of the rocket caused the third stage to separate early. It meant that the payload, the world's first geostationary scientific satellite known as Geos, could not achieve its planned orbit and that seven experiments which took years to prepare might no longer be done.

In fact the despair looks as though it may have been premature. For although the engineers from the rocket manufacturers McDonnell Douglas gathered in California to discuss the Delta's acknowledged failure, the scientists who met at the ESA's Space Operations Centre at Darmstadt in West Germany did so a little more optimistically. On the previous day Geos had been placed in an elliptical orbit whose apogee, at $38,498 \mathrm{~km}$, was easily as high as the originally intended circular orbit.

The difference, of course, was that the new orbit, not heing geostationary, would not allow the satellite to be permanently within sight of the Darmstadt ground station. It is understood, however, that not all the data obtained over each 24-hour period would have been used anyway. The new elliptical orbit, selected to maximise the return of scientific data, has a perigee of $2,131 \mathrm{~km}$ and an inclination of $26.85^{\circ}$. It is traversed every 12.06 hours by the satellite, which could now be within sight for some 10 hours a day.

As the scientists gathered to decide which experiments to pursue and data to collect, ESA officials were pleased to have retrieved, if only partially, a mission which at first looked on the very edge of disaster. The spacecraft and third stage assembly should have spun up to 97 revolutions per minute before separating from the second stage. This spin stabilisation would have ensured that the third stage had an accurate trajectory during its burn; without it, the spacecraft tumbled around into the wrong orbit, though it was stabilised soon thereafter.

The spacecraft could not have been allowed to stay indefinitely in this socalled transfer orbit because it went through zones of high radiation which might endanger the experiments and decrease the total active lifetime of the spacecraft. To stay in that orbit also served no useful purpose: it was not on the magnetic field lines connected to the auroral zones of the Arctic and Antarctic, and the experiments would probably not work with the field and particle intensities so high.

The original intention was that the spacecraft should be injected into an elliptical transfer orbit (apogee 35,500 $\mathrm{km}$, perigee $230 \mathrm{~km}$ ) and then be boosted on the fourth apogee to a circular orbit synchronised with the Earth below. That the European-built solid-propellant apogee booster motor responsible for this task placed Geos in the elliptical orbit it now occupies is a cause for comfort, even though the motor could not be used twice to achieve a circular orbit. But then so much could so easily have gone wrong.

The effect, ironically, is that Geos is now placed in a fashion that had been expected of the ESA's spare 'qualification' geosynchronous satellite which was due to be sent up in 1979 atop a European Ariane launcher now being developed. It remains possible that this back-up satellite will be upgraded to become the 'true Geos', but it will cost the ESA another 13 million units of account to purchase the launch from NASA - a cost which is not recoverable for last week's launch under NASA's no-loss-no-profit contracts. Although the ESA Council is due to meet next week, it is unlikely to discuss this idea of inverting the roles of the two satellites.

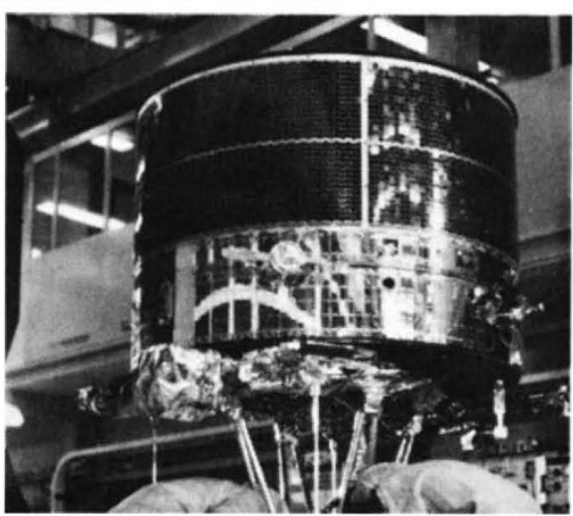

